\title{
Détermination De La Dose Optimale D'engrais Minéral 15-15-15 Sur Cinq (05) Variétés De Maïs Doux (Zea Mays L. ssp. saccharata) Au Sénégal
}

\section{Mariama Dalanda Diallo, PhD Ä̈chatou Toure}

Section Productions Végétales et Agronomie, UFR des Sciences Agronomiques, de l'Aquaculture et des Technologies Alimentaires,

Université Gaston-Berger, Saint-Louis, Sénégal

\section{Fatou Diop Mbacke}

Centre pour le développement de l'horticulture $(C D H)$, Institut sénégalais de recherches agricoles (ISRA), Cambérène, Dakar, Sénégal

\section{Minda Mahamat Saleh, PhD}

Département de Biologie Végétale, Faculté des Sciences et Techniques,

Université Cheikh Anta Diop, Dakar-Fann, Sénégal

\section{Goalbaye Touroumgaye, PhD}

Institut Universitaire des Sciences Agronomiques et de l'Environnement (IUSAE-S) BP 105 Sarh, Tchad

\section{Ahmadou Bamba Ndiaye}

Nafi Diop Ndiaye

Institut de Technologie Alimentaire (I.T.A.), Route des Pères Maristes, Dakar Hann, Sénégal

\section{Aliou Diop, Prof.}

Section Mathématiques Appliquées, UFR des Sciences Appliquées et

Technologie, Université Gaston-Berger, Saint-Louis, Sénégal

Aliou Guisse, Prof.

Département de Biologie Végétale, Faculté des Sciences et Techniques,

Université Cheikh Anta Diop, Dakar-Fann, Sénégal

doi: 10.19044/esj.2016.v12n27p135 URL:http://dx.doi.org/10.19044/esj.2016.v12n27p135

Abstract

The objective of this study is to assess the response of five varieties of sweet maize namely, Prime plus (V1), Shy 1036 (V2), Colombus (V3), GSS (V4), and JKMH-45 (V5) to three different doses of NPK (15-15-15) fertilizer: F1 (normal dose), F2 (75\% of the normal dose) and F3 (125\% of the normal dose). An experimental design in Fisher blocs with 15 treatments was established at the ISRA/CRA experimental station in Saint Louis 
(Senegal). The results have shown that 30 days after sowing (DAS), the varieties Colombus, GSS and JKMH-45 have had the highest heights with $32.75 \mathrm{~cm}, 31.85 \mathrm{~cm}$ and $31.46 \mathrm{~cm}$, respectively with the F2 dose. The variety JKMH-45 has a greater height of the upper ear $(89.39 \mathrm{~cm})$ at 60 DAS and valorizes better the F2 dose (75\% of the normal dose). The F3 dose increases less the plant's height. A non-significant difference was observed among the 5 varieties used regarding the precocity. The variety V2 or Shy 1036 developed ears at first, but is not precocious compared to the variety V1 that not only shows earlier anthesis but also an appearance of darker hairs. The variety JKMH-45, although presenting darker hairs after the variety Prime plus, is the latest variety, earing at 57 DAS and with male and female anthesis appearing at 59 DAS and 63 DAS. This study can constitute a basic criterion in differentiating the sweet maize varieties in Senegal. It would also allow more financial saving on inputs and contribute to the maximization ofproduction if the choice of the variety is well done.

Keywords: Sweet Maize, Mineral Fertilizer, Fertilization, Ndiol, Senegal River valley

\section{Résumé}

L'objectif de cette étude est d'évaluer le comportement de cinq variétés de maïs doux (Prime plus (V1), Shy 1036 (V2), Colombus (V3), GSS(V4), JKMH-45 (V5)) vis-à-vis de trois doses d'engrais 15-15-15 différentes (F1 la dose normale ; F2 75\% de la dose normale et F3 125\% de la dose normale). Un dispositif expérimental en bloc de Fisher avec 15 traitements a été mis en place au niveau de la station expérimentale de l'ISRA/CRA de Saint-Louis. Les résultats ont montré qu'à 30 jours après semis, les variétés Colombus, GSS et JKMH-45 ont eu les plus grandes hauteurs soit respectivement $32,75 \mathrm{~cm} ; 31,85 \mathrm{~cm}$ et $31,56 \mathrm{~cm}$ avec la dose F1. La variété JKMH-45 présente une plus grande hauteur d'insertion de l'épi supérieur soit $89,39 \mathrm{~cm}$ à 60 jours après semis et valorise mieux la dose F2 (75\% de la dose supposée normale). Les doses F3 augmentent moins la hauteur des plants. Une différence non significative a été décelée entre les 5 variétés utilisées en ce qui concerne la précocité. La variété V2 ou Shy 1036 a épié en premier mais n'est pas précoce par rapport à la variété V1 Prime plus qui non seulement présente une anthèse plus hâtive mais également à l'aspect de la soie plus foncé. La variété JKMH-45, bien qu'ayant des soies plus sombres après la variété Prime plus, constitue la variété la plus tardive avec une épiaison à $57 \mathrm{JAS}$ et des anthèses mâle et femelle respectivement à 59 JAS et à 63 JAS. Cette étude peut constituer un critère de base pour différentier les variétés de maïs doux au Sénégal. Elle permettrait aussi 
d'économiser en termes de finance sur les intrants apportés et de maximiser le rendement si le choix de la variété est bien fait.

Mots-clés : Maïs Doux, Fumure Minérale, Fertilisation, Ndiol, Vallée du Fleuve Sénégal

\section{Introduction}

Le maïs (Zea mays L.) est la plante la plus cultivée au monde et la première céréale produite devant le blé (Tahir et al., 2009 ; Missihoun et al., 2012). Au Sénégal, il est principalement cultivé dans les zones Centre, Sud et Est du Sénégal (Sine-Saloum, Casamance et Sénégal Oriental). Cette céréale est également cultivée dans la Vallée du Fleuve Sénégal, essentiellement lors des périodes de décrue (ISRA, 2008). La production du maïs prend de l'ampleur en rapport avec les multiples usages dont il est l'objet : consommation humaine, consommation animale et biocarburant et les incitations directes ou indirectes dont il a bénéficié (Soule and Gansar, 2010). Le maïs est devenu la deuxième céréale la plus produite dans le pays. Son niveau de production représente $22 \%$ de la production céréalière nationale (Ndiaye \& Niang, 2010). En ce qui concerne le Maïs Doux, le Sénégal est le deuxième fournisseur du marché européen derrière le Maroc, avec une production de près de 7000 tonnes. Avec un tonnage nul en 2006, la production locale exportée est passée de 260 à 4435 t entre 2007 et 2010 (Dia, 2012). C'est ainsi qu'étant identifié comme une spéculation à fort potentiel notamment à l'export et dont la mise en œuvre permettra la promotion et la création d'entreprises productrices et transformatrices, le Maïs Doux a été classé parmi les créneaux porteurs du secteur primaire d'après la Direction de l'Appui du Secteur Privé (Dia, 2012). Au Sénégal, le circuit de distribution du Maïs Doux est constitué uniquement par les conserves importées de France ou d'Asie. D'après la FAO (2010), les quantités importées sont respectivement de 253, 329 et 185 t entre 2007 et 2009. Ainsi, au moment où la plus grande production est exportée, la demande nationale est entièrement couverte par les conserves importées (Ndiaye, 2007). Donc, il y a lieu de réfléchir à une démarche visant à user des opportunités offertes pour favoriser la culture et la transformation de maïs doux dans le but de répondre à la demande nationale. Pour y parvenir, il est important de déterminer le potentiel agronomique et les propriétés physico-chimiques de quelques cultivars de maïs doux. La nécessité d'améliorer les rendements et la productivité de cette culture devient alors un objectif primordial. Cela peut se faire par l'amélioration des techniques culturales comme l'apport d'engrais organiques et chimiques adéquats et la sélection de variétés plus productives. Selon Nyembo (2010), l'utilisation de variétés améliorées et le recours à la fertilisation minérale, augmentent les 
rendements de l'ordre de 4000 à $6000 \mathrm{Kg} / \mathrm{ha}$.

C'est dans cette optique que notre étude a été mis en place à Ndiol, dans la région de Saint Louis au Nord du Sénégal, avec comme objectif d'évaluer le comportement de cinq variétés de maïs doux (Prime plus (V1), Shy 1036 (V2), Colombus (V3), GSS(V4), JKMH-45 (V5)) vis-à-vis de trois doses d'engrais 15-15-15 différentes (F1 la dose normale ; F2 75\% de la dose normale et F3 $125 \%$ de la dose normale). Il s'agit de déterminer de la dose optimale d'engrais chimique et d'identifier de la variété de maïs doux qui réagit le mieux à la fertilisation minérale. Notre hypothèse est qu'une augmentation de la dose de 15-15-15 augmenterait la productivité du maïs et qu'aux mêmes doses d'engrais chimiques, les variétés réagiraient différemment.

\section{Matériel et Méthodes}

\section{Le site d'étude}

Les essais ont été mis en place à la station de Recherche Expérimentale Agricole de l'ISRA/CRA de Saint Louis située à l'entrée du village de NDIOL (16 $6^{\circ} 8^{\prime} 22^{\prime \prime}$ Nord et $16^{\circ} 19^{\prime} 5^{\prime \prime}$ Ouest, altitude $7 \mathrm{~m}$ ) (Figure 1). La station a été créée en 1977 et se situe à $28 \mathrm{Km}$ de la ville de Saint- Louis sur la route de Richard Toll La zone est caractérisée par un climat chaud et sec avec une alternance de deux saisons : une saison pluvieuse concentrée sur trois mois (juillet, août et septembre) et une saison sèche de neuf mois (octobre à juin). La pluviométrie est faible et dépasse rarement $400 \mathrm{~mm} / \mathrm{an}$. La température moyenne annuelle est de $28^{\circ} \mathrm{C}$ avec un maximum de $31,9{ }^{\circ} \mathrm{C}$ et un minimum de $15,2{ }^{\circ} \mathrm{C}$. Dans la zone du delta du fleuve Sénégal, l'apport des pluies d'hivernage n'est que de 15 à $20 \%$ de la consommation du riz (Le Brusq and Loyers, 1982).

La station d'étude est caractérisée par deux types de sols. Les sols "walo" ou hollaldés sur lesquels des essais de criblage de variétés de riz sur la tolérance à la salinité sont effectués. Ce sont des vertisols dont la teneur en argile dépasse $60 \%$. Ensuite, les sols "'diéri', sableux, pauvres et secs, sur lesquels on retrouve des essais sur les cultures maraichères comme le cas de notre essai de maïs doux, mais également fruitières et sur les grandes cultures. La multiplication de semences (arachide, niébé, blé et la culture de Jatropha curcas occupe la majeure partie des activités. Avant la mise en place de la culture, la parcelle était en jachère (Michel, 1973).

\section{Matériel biologique}

Les cultivars de maïs doux évalués dans notre expérimentation ont été au nombre de 5. Les informations relatives à ces cultivars sont confinées dans le Tableau 1. 


\section{Fertilisants}

L'Urée (46\% d'azote), du fumier de cheval à la dose 40 t/ha et de l'engrais minéral 15-15-15 à différentes doses ont été utilisés comme fertilisants.

\section{Dispositif expérimental}

Le dispositif expérimental est en bloc aléatoire complet (BAC) ou bloc de Fisher avec deux facteurs : dose de fertilisation à trois modalités (F1, F2 et F3) et variété à cinq modalités (V1, V2, V3, V4 et V5). Le nombre répétition a été de 04 et la combinaison des modalités des deux facteurs avait donné 15 traitements (Tableau 2).

Les dimensions des parcelles élémentaires sont de $3 \mathrm{~m}$ sur $5 \mathrm{~m}$. Les blocs ont une longueur de $59 \mathrm{~m}$ et $26 \mathrm{~m}$ de large. Dans chaque répétition du bloc, nous avons $(5 * 3)$ soit 15 parcelles élémentaires avec des allées de $1 \mathrm{~m}$ entre les parcelles élémentaires et $2 \mathrm{~m}$ entre les blocs. La superficie d'une parcelle élémentaire est de $15 \mathrm{~m}^{2}$ et la superficie totale de l'essai est de 1534 $\mathrm{m}^{2}$ avec les allées (Figure 2 ).

La préparation du terrain s'est effectuée par un labour suivi d'un offsetage croisé pour ameublir le sol à une profondeur de $25 \mathrm{~cm}$. En fumure de fond, il a été épandu du fumier de cheval à la dose 40 t/ha et de l'engrais minéral 15-15-15 a été apporté à différentes doses :

$\mathrm{F} 1$ : DN $200 \mathrm{~kg} / \mathrm{ha}$ soit $330 \mathrm{~g} /$ parcelle élémentaire

$\mathrm{F} 2$ : $75 \% \mathrm{DN}$ soit $165 \mathrm{~kg} / \mathrm{ha}$ donc $247,5 \mathrm{~g} /$ parcelle élémentaire

F3 : 125\% DN soit $220 \mathrm{~kg} /$ ha donc $412,5 \mathrm{~g} /$ parcelle élémentaire

Pour le traitement du sol, du Mocab a été épandu 5 à la dose de 5 $\mathrm{g} / \mathrm{m}^{2}$. Le semis direct du maïs doux a été effectué deux semaines après. C'est un semis à plat à deux graines par poquet. Chaque parcelle élémentaire a comporté 5 lignes espacées de $75 \mathrm{~cm}$ sur $3 \mathrm{~m}$. Sur chaque ligne les poquets ont été espacés de $25 \mathrm{~cm}$ sur $5 \mathrm{~m}$ de long. Après le semi, une fertilisation a été effectuée par l'apport d'une fumure de couverture, consistant à épandre de l'urée à la dose de $200 \mathrm{~kg} /$ ha en deux phases à $30 \mathrm{JAS}$ et à $45 \mathrm{JAS}$ soit $50 \%$ des besoins à chaque phase. Pour le traitement phytosanitaire, du Fongex a été appliqué sur la parcelle expérimentale à la dose de $10 \mathrm{~g} / 1$ à 39 JAS, ensuite de l'Acéphale à la dose $5 \mathrm{cc} / 1$ à 59 JAS et du Deltaméthrine à la dose $10 \mathrm{cc} / 1$ à 74 JAS pour le contrôle des insectes défoliateurs et des borers de tiges. Le contrôle des mauvaises herbes a été effectué par la suite par sarclages à la houe autant que nécessaire. La récolte a été effectuée à 90 JAS. Les données ont été collectées de manière aléatoire sur les 3 lignes centrales de chaque parcelle élémentaire avec des sauts de 2 plants sur les extrémités avant et arrière de chaque ligne. Les épis sont mis dans des sachets, étiquetés puis amener au laboratoire pour peser, triage et analyse. Les variables étudiées au cours de la culture sont: la hauteur à $30 \mathrm{JAS}$, le taux 50\% de 
floraison, la hauteur d'insertion de l'épi supérieur, l'aspect de la soie à 60 JAS, le nombre d'épi par plant et le nombre de grains par épi.

\section{Analyse statistique}

L'analyse de variance (ANOVA) a été effectuée avec le logiciel XLSTAT version 2010. Le test de Fisher LSD a été utilisé pour la comparaison des moyennes au seuil de $5 \%$.

\section{Résultats}

\section{Hauteur 30 JAS}

L'ANOVA de la hauteur des plants à 1 mois après semis montre une différence significative suivant la dose de fertilisation et non significative suivant la variété (Figure 3). La plus grande hauteur a été obtenue avec la dose F1 (DN) contrairement à la dose F3 $(125 \% \mathrm{DN})$ qui a la plus petite valeur soit de classe B. L'interaction Dose-Variété aussi présente aussi une différence significative. La variété Colombus associée à la dose F1 présente une meilleure hauteur contrairement à la variété Shy 1036 associé à la même dose.

\section{Hauteur d'insertion de l'épi supérieur}

L'analyse de la variance de la hauteur d'insertion de l'épi supérieur montre une différence significative en fonction des variétés (Figure 4). La variété JKMH-45 présente une plus grande hauteur d'insertion contrairement à la variété Prime plus avec la plus petite valeur.

L'interaction de la dose et la variété montre aussi une différence significative avec une hauteur d'insertion maximale de la variété JKMH-45 associée à la dose F2. L'interaction de la variété Shy 1036 et la dose F1 donne la plus petite hauteur d'insertion.

\section{$\mathbf{5 0 \%}$ Floraison}

L'analyse de la Figure 5 montre que la variété JKMH-45 a tardivement eu les fleurs males apparues sur plus de $50 \%$ de ses plants soit à 59 JAS contrairement à la variété Prime plus qui atteint les $50 \%$ dès la $38^{\mathrm{eme}}$ JAS.

Pareillement pour la floraison male, l'analyse des 50\% de floraison femelle ou ébauches des premières soies montre aussi les tendances selon lesquelles la variété JKMH-45 (V5) est la dernière à avoir plus de 50\% des plants avec des soies, soit à 63 JAS et la variété prime plus la première à l'atteindre à la date du $50 \mathrm{JAS}$. Cependant, aucune différence significative n'a été décelée ni suivant la dose, ni suivant la variété au seuil de $5 \%$. 


\section{Aspect de la soie à $60 \mathrm{JAS}$}

L'ANOVA de l'aspect de la soie à 60 JAS (Figure 6) révèle une différence significative en fonction de la variété et de l'interaction des facteurs dose et variétés. En ce qui concerne les variétés, Prime plus a une soie plus colorée à cette date au moment où les variétés JKMH-45, GSS et Colombus ont une moins colorée.

L'interaction montre que la dose $\mathrm{F} 1(\mathrm{DN})$ appliquée à la variété Prime plus présente une soie plus colorée (brune) alors que la dose F3 (125\% DN) appliquée à la variété Colombus donne une soie plus claire (blanchâtre).

\section{Le nombre d'épis par plant}

L'analyse de la variance en Fisher (LSD) du nombre d'épis moyen par plant (Figure 7) montre une différence significative en fonction des variétés. La variété Prime plus présente un plus grand nombre d'épis par plant soit 1,51 en moyenne contrairement à la variété JKMH-45 qui n'en présente que 1,14 en moyenne.

L'interaction de la variété et la dose également présente une différence très significative. La variété Colombus associée à la dose F2 $(75 \% \mathrm{DN})$ donne un plus grand nombre d'épis. La variété JKMH-45 cependant, associée à la dose la plus grande donne moins d'épis par plant soit un nombre de 1 .

\section{Nombre de grains par épi}

L'analyse de la variance du nombre de grains par épis (Figure 8) montre une différence significative en fonction du facteur variété. Les variétés JKMH-45 (V5) et Colombus (V3) ont le plus grand nombre de grains par épi avec la V5 qui a le maximum soit vers 433 grains. La variété Prime plus (V1) quant à elle, a un plus petit nombre de grains par épi soit vers 304 grains.

L'analyse de l'interaction des deux facteurs révèle aussi que la V5 associée à la dose F2 et à la F3 donne un nombre de grain élevé qui tourne vers 446 grains contrairement à la variété Prime plus associée à la dose F1.

\section{Discussion}

L'étude que nous avons effectuée à Ndiol et qui a porté sur cinq variétés de maïs doux à savoir, Prime plus (V1), Shy 1036 (V2), Colombus (V3), GSS (V4), JKMH-45 (V5) nous a permis de déceler une très grande différence entre les variétés. En ce qui concerne la hauteur, à 30 jours après semis, les variétés Colombus, GSS et JKMH-45 ont eu les plus grandes hauteurs La variété JKMH-45 présente une plus grande hauteur d'insertion de l'épi supérieur et valorise mieux la dose F2 soit 75\% de la dose supposée normale. Les doses F3 augmentent moins la hauteur des plants. Nous 
pouvons donc dire qu'un surdosage de l'engrais minérale 15-15-15 sur le maïs doux ne favorise pas une plus grande hauteur. Selon Kabrah et al. (1996) et Useni et al. (2012), l'excès d'azote entraine une croissance végétative exagérée et un retard de la maturité comme un retard ou une absence de floraison (dû à l'allongement excessif de la période végétative. En ce qui concerne la précocité des variétés, une différence non significative a été décelée ce qui veut dire que les 5 variétés utilisées ont une différence de précocité pas très importante. Par ailleurs, l'analyse montre que la variété V2 ou Shy 1036 a épié en premier mais n'est pas précoce par rapport à la variété V1 Prime plus qui non seulement présente une anthèse plus hâtive mais également à l'aspect de la soie plus foncé, ce qui traduit le fait qu'elle est présentée en premier des soies. La variété JKMH-45, bien qu'ayant des soies plus sombres après la variété Prime plus, elle constitue la variété la plus tardive avec une épiaison à 57 JAS et des anthèses mâle et femelle respectivement à $59 \mathrm{JAS}$ et à $63 \mathrm{JAS}$. Ces résultats confirment ceux trouvés par Kidinda et al (2015) et Delsoin (2014) qui ont montré que la dose d'engrais minérale 15-15-15 n'influe en rien sur la floraison du maïs doux. De même que les écarts entre les anthèses mâles et femelles sont phénologiques.

En ce qui concerne les rendements, caractérisé ici par le nombre d'épi par plant, critère le plus important pour le choix d'une variété et d'une dose de fertilisation, il y a une différence significative entre les traitements. Les variétés de plus petite taille présentent plus d'épi par plant à l'image de la variété Colombus et Prime plus. Mais celles qui présentent moins d'épi ont un plus grand poids de l'épi. Ce qui veut dire que la plante polarise tous ses éléments sur un seul épi au lieu de les repartir dans deux épis comme la variété Prime plus qui a plus d'épi et moins de masse. La dose F2 est celle qui donne plus d'épis par plant avec la variété Colombus et un plus grand poids avec la variété JKMH-45. Selon plusieurs études, l'effet de la fertilisation azotée sur la productivité des cultures sont très variables et les apports d'azote enlevées peuvent aussi provoquer la verse de la plante et ainsi réduire les rendements engrains (Hauck, 1990 ; Lafond, 2004). Des études menées par Batiano et al. (2004) ont montré l'effet significatif de la fertilisation chimique sur le maïs et les travaux de Nyembo et al. (2012) ont montré que la chute du rendement observé avec les doses d'engrais minéraux les plus élevées, montre que la phase de remplissage des grains est un stade déterminant dans l'élaboration du rendement et qu'elle aurait été perturbée. Par contre d'autres études ont montré le contraire. Diepenbrock et al. (1995) ont obtenu des rendements en grains plus élevés avec une augmentation de la dose de la fertilisation azotée. Grant et al. (1999), qui ont obtenu des augmentations de rendements suite à l'application d'engrais azotés, ont 
indiqué également que l'application d'azote entrainait plutôt une réduction des rendements en grains lorsque la verse était présente.

\section{Conclusion}

Le maïs doux, culture horticole d'exportation est véritablement reconnue pour générer des emplois et participer à la sécurité alimentaire de notre pays si la production est destinée au marché local. Donc, des études comme celle-ci demeure intéressante pour caractériser différentes variétés combinées à la meilleure dose de fertilisation minérale afin de pouvoir en aval les vulgariser vers les producteurs. Ainsi, au terme de notre expérimentation, les variétés JKMH-45 et Colombus ont été notées comme étant les plus performantes. En termes de dose de fertilisation, la plus petite à savoir la F2 $(75 \%$ DN) s'avère être la meilleure et l'hypothèse selon laquelle une augmentation de la dose de 15-15-15 augmenterait la productivité du maïs n'est pas vérifiée. Ceci permettrait d'économiser en termes de finance sur les intrants apportés et de maximiser le rendement.

\section{Remerciements}

Les auteurs tiennent à remercier projet de l'USAID/ERA pour le soutien financier apporté à cette étude. Ils remercient également le Projet d'Appui à la Promotion des Enseignantes-chercheures du Sénégal (PAPES) du Ministère de 1'Enseignement Supérieur et de la Recherche pour les frais de publication.

\section{References:}

Bationo A., Kimetu J., Ikera S., Kimani S., Mugenda D., Odendo M., Silver M., Swift M.J., Sanginga N. The Africa Network for soil biology and fertility: New challenge and opportunities, in Bationo (Ed): Managing of Nutrient Cycles to sustain soil fertility in Sub-Saharan Africa. Academy of science publishers, Nairobi, Kenya, 1-23p, 2004.

Dia M.L. impact des nouvelles normes sanitaires sur la structure de la filiere d'exportation horticole sénégalaise, Thèse de Doctarat Unique en Sciences Économiques, UCAD, 231 P., 2012.

Diepenbrock W.A., Léon J., Clasen K., Yielding ability and yield stability of linseed in Central Europe, Agronomy Journal ,87, 84-88, 1995.

FAOSTAT. Food and Agriculture Organization of the United Nations. Apperçu du développement Rizicole, Sénégal, Brochure produite par la division de la production végétale et de la protection des plantes (PDP), $10 \mathrm{p}$, 2010.

Grant C.A., Dribnenki J.C.P., Bailey L.D. A comparison of the yield response of solin (cv. Linola 947) and flax (cvs. McGregor and Vimy) to application of nitrogen, phosphorus, and Provide (Penicillium bilaji), 
Canadian Journal of Plant Sciences, 79, 527-533, 1999.

Hauck R.D. Agronomic and public aspects of soil nitrogen research, Soil Use Management, 6, 66-70, 1990.

ISRA. État des ressources phytogénétiques pour l'alimentation, et l'agriculture dans le monde, Second rapport général du Sénégal, 45 p., 2008. Kidinda K.L., Kiluba K.J., Tshipama T.D., Kilumba K.M., Mpoyo M.G.l., Langunu S., Muteba K.M.l, Nyembo K.L. Mise en évidence des doses de fertilisants minéraux à appliquer aux nouvelles variétés de maïs introduites dans la région de Lubumbashi (RD Congo), International Journal of Innovation and Applied Studies, 12, 1, 96-103, 2015.

Lafond J. Fractionnement de la fertilisation azotée minérale et organique: Effet sur la productivité du canola de printemps et sur les nitrates du sol, Canadian Journal of Soil Science, 84, 491-501, 2004.

Le Brusq, J.Y., Loyers, J.Y. Evolution de la salinité des sols et des eaux en relation avec la riziculture submergée dans le delta du fleuve Sénégal : résultats de la campague de 1981. ORSTOM, Dakar (SEN), 45p., 1982.

Michel P. Les bassins des fleuves Sénégal et Gambie : étude géomorphologique. Thèse. Mémoire Orstom, 3 tomes, 753 p., 1973

Missihoun A.A., Agbangla C., Adoukonou-Sagbadja H., Ahanhanzo C., Vodouhè R. Gestion traditionnelle et statut des ressources génétiques du sorgho (Sorghum bicolor L. Moench) au Nord-Ouest du Bénin, International Journal of Biological Chemical Sciences, 6, 1003-1018, 2012.

Ndiaye M. Senegal Agricultural Situation: Country Report 2007. Gain Report Number SG7001 Washington, DC: Service chargé de l'agriculture étrangère (FAS) du Ministère de l'Agriculture des États-Unis (USDA), 2007. Ndiaye M., Niang M., De l'étude sur la transmission des fluctuations et le calcul de prix de parité à l'importation/exportation dans la sous-région: Cas pratique du Sénégal (Rapport final). CSA-SIM, Dakar, Sénégal. 50 p., 2010.

Nyembo K.L. Augmentation du rendement du maïs par l'exploitation de l'effet hétérosis des hybrides produits au Katanga, République Démocratique du Congo, Thèse de doctorat, Faculté des sciences agronomiques, Université de Lubumbashi, 157 p., 2010.

Nyembo K.L., Useni S.Y., Mpundu M.M., Bugeme M.D., Kasongo L.E., Baboy L.L. Effets des apports des doses variées de fertilisants inorganiques sur de Zea mays. Journal of Applied Biosciences, 59, 4286- 4296, 2012.

Tahir M., Javed M. R., Tanveer A., Nadeem M.A., Wasaya A., Bukhari S.A.H., Rehman J.U. Effect of different herbicideson weeds, growth and yield of spring planted maize (Zea mays L.), Pak. J. Life Soc. Sci, 7(2), 168174, 2009.

Useni S.Y., Baboy L.L., Nyembo K.L., Mpundu M.M. Effets des apports combinés de biodéchets et de fertilisants inorganiques sur le rendement de trois variétés de Zea mays L. cultivées dans la région de Lubumbashi, 
Journal of Applied Biosciences, 54, 3935- 3943, 2012.

\section{Liste des tableaux}

Tableau 1 : Cultivars de l'expérimentation

\begin{tabular}{cccc}
\hline Variétés & Codes & Couleur & Origine \\
\hline Prime plus & V1 & Jaune & Top Mountain \\
Shy 1036 & V2 & Jaune & Syngenta seed-SCL \\
Colombus & V3 & Jaune & Syngenta seed-SCL \\
GSS & V4 & Jaune & Syngenta seed-SCL \\
JKMH-45 F1 & V5 & Jaune & Traoré et fils \\
\hline
\end{tabular}

Tableau 2: Tableau des traitements

\begin{tabular}{cccc}
\hline \multirow{2}{*}{ Variété } & \multicolumn{3}{c}{ Dose d'engrais } \\
\cline { 2 - 4 } & F1 & F2 & F3 \\
\hline V1 & V1 F1 & V1F2 & V1F3 \\
V2 & V2 F1 & V2F2 & V2F3 \\
V3 & V3 F1 & V3F2 & V3F3 \\
V4 & V4 F1 & V4F2 & V4F3 \\
V5 & V5 F1 & V5F2 & V5F3 \\
\hline
\end{tabular}

\section{Liste des Figures}

Figure 1 : carte de localisation du site de Ndiol (Région de Saint Louis)
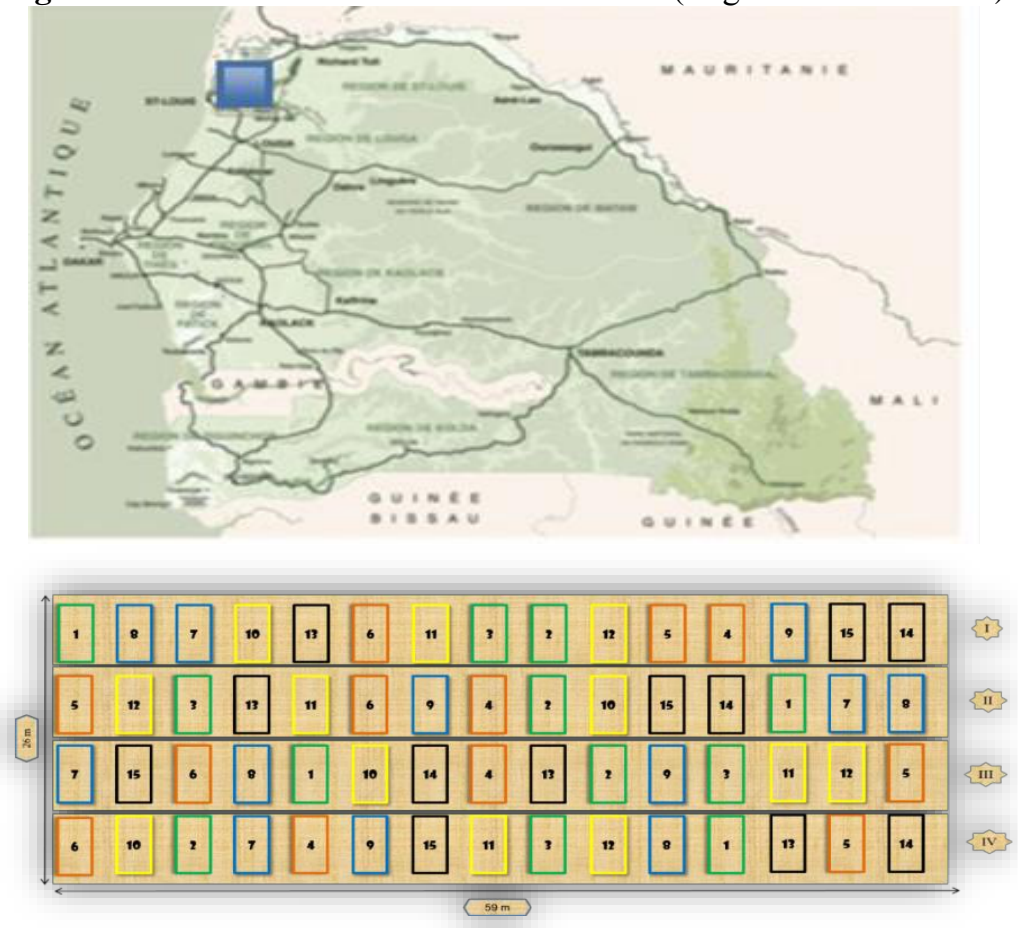

Figure 2: Dispositif expérimental mis en place 


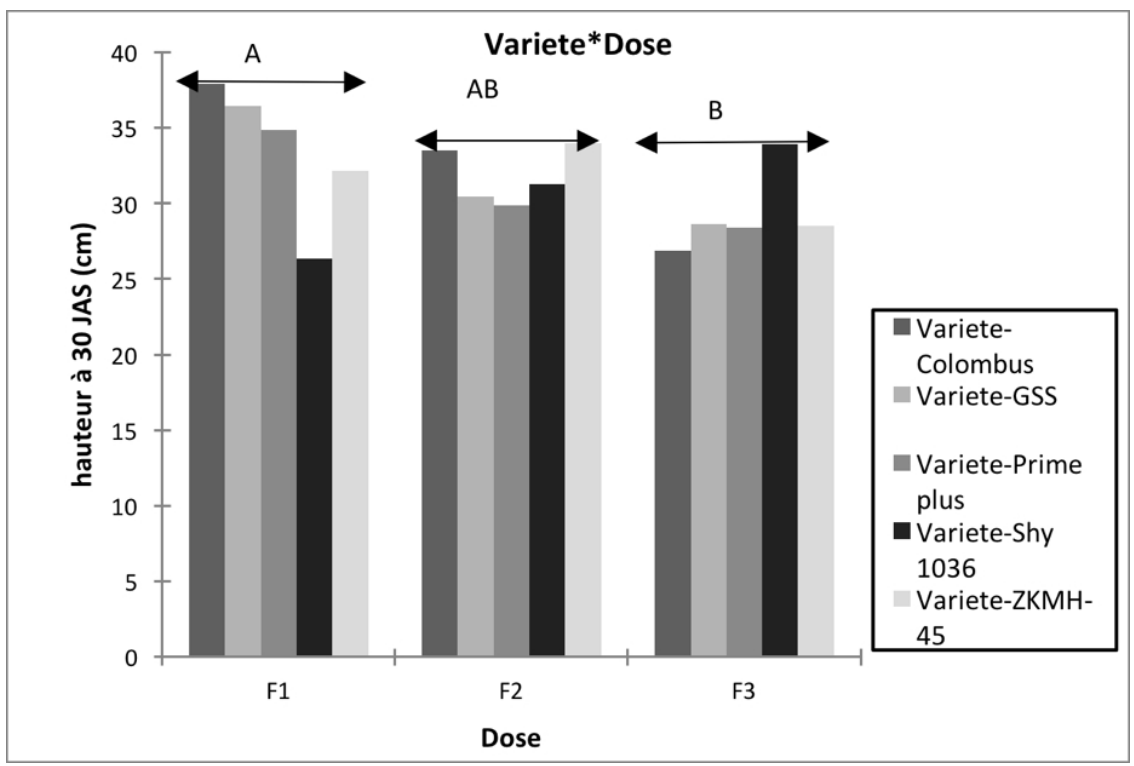

Figure 3: hauteur à $30 \mathrm{JAS}$

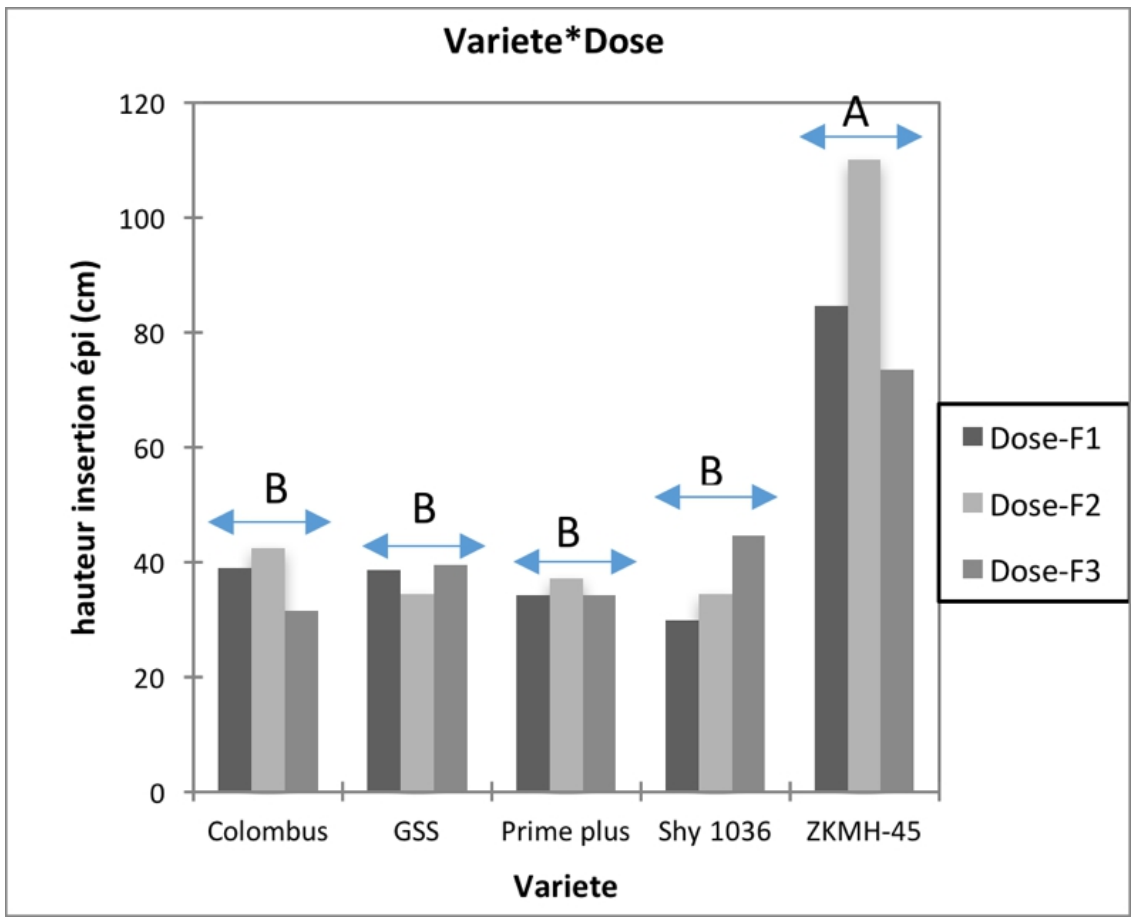

Figure 4: hauteur d'insertion de l'épi 


$$
100
$$

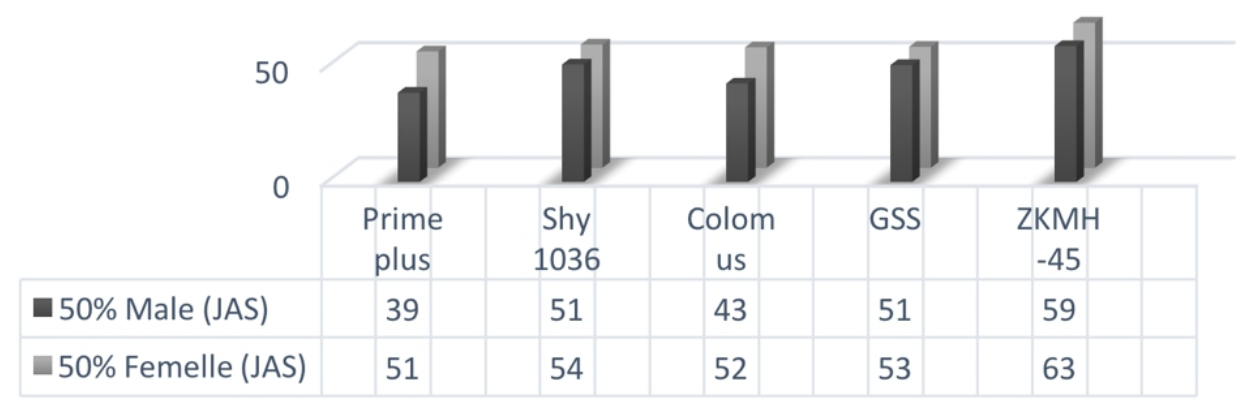

- $50 \%$ Male (JAS) $\quad 50 \%$ Femelle (JAS)

Figure 5: 50\% floraison mâle et femelle

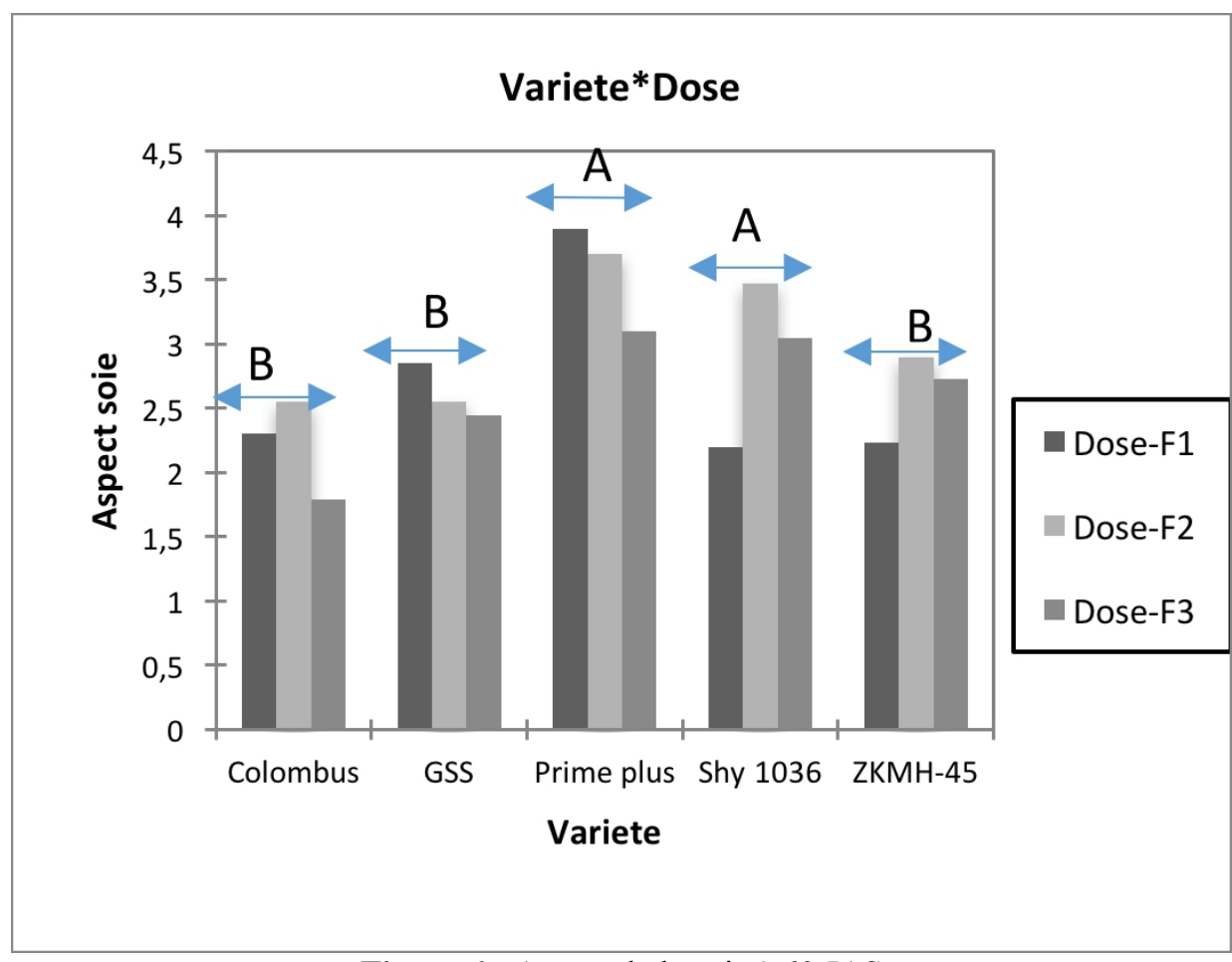

Figure 6 : Aspect de la soie à $60 \mathrm{JAS}$ 


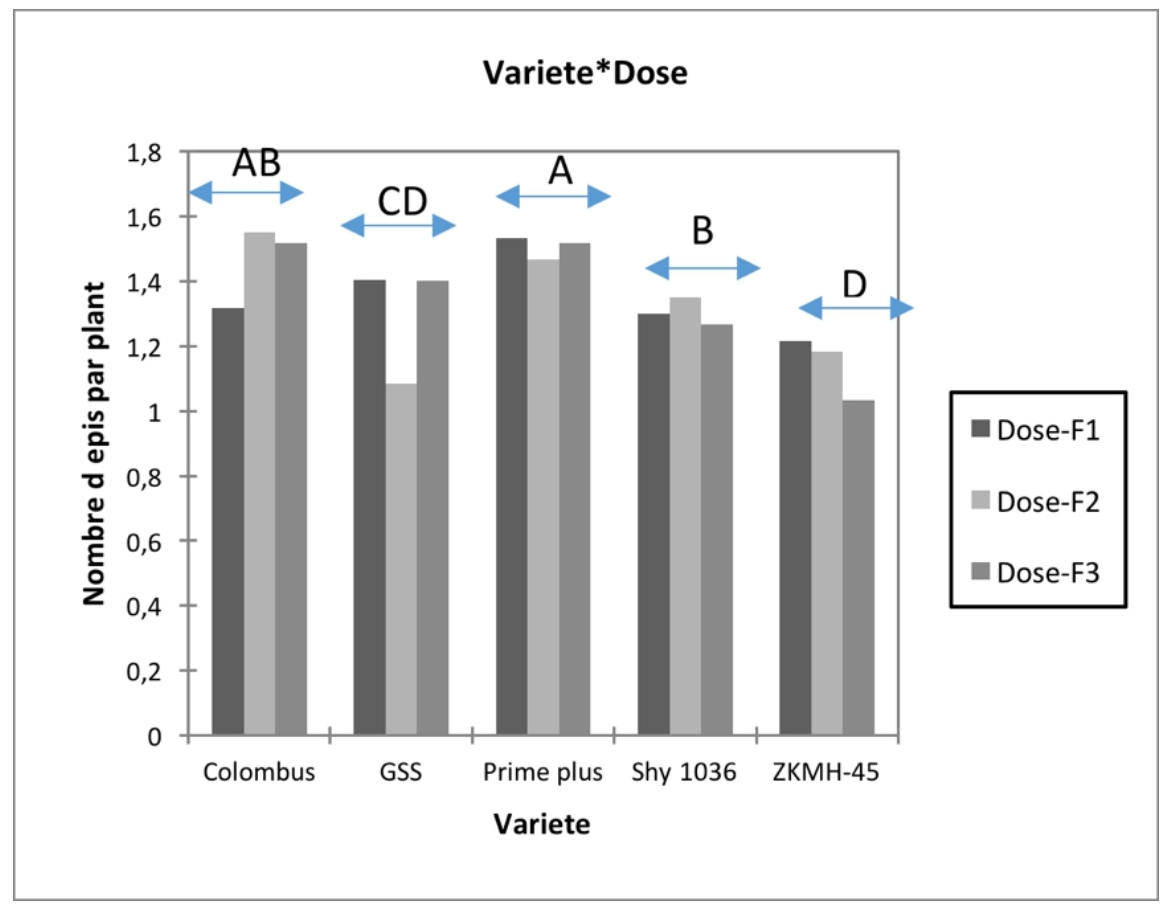

Figure 7: nombre d'épis par plant

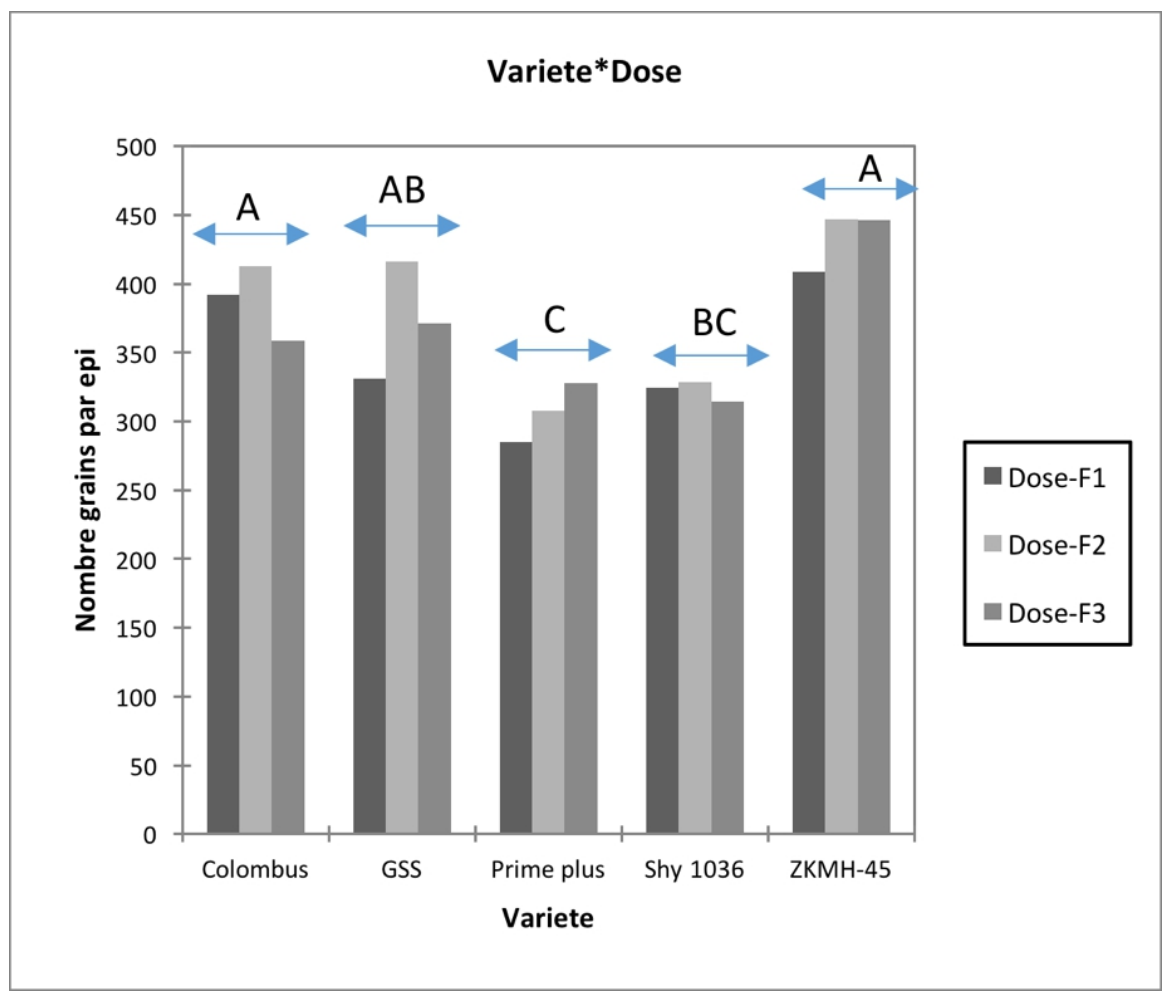

Figure 8: Nombre de grains par épi 